\title{
Sintomas e sinais: uma abordagem científica do exame clínico
}

António Vaz Carneiro*

A evolução da medicina ao longo dos últimos 100 anos tem sido uma história de sucesso indiscutível. A vertente tecnológica médica tem tido uma evolução verdadeiramente impressionante, com novas técnicas de imagem, novas metodologias diagnósticas analíticas e novas abordagens terapêuticas, farmacológicas ou outras.

Apesar de toda esta parafernália, o exame clínico (EC) - constituído pela história e pelo exame objectivo - mantém-se a base de todo o acto médico, especialmente no que concerne a área do diagnóstico.

Durante décadas, o EC foi tido como o centro da «arte» médica, um procedimento envolvido em mistério e passado de geração em geração através dos ensinamentos dos mais «velhos» (i.e., mais experientes). Ainda nos lembramos dos grandes clínicos diagnosticadores dos anos 50, 60, 70, médicos de enorme prestígio, precisamente porque possuíam a chave da descoberta da doença através de um EC minucioso, cuidadoso e detalhado e um raciocínio clínico muito apurado.

A irrupção de novas técnicas diagnósticas veio confirmar, mas também anular, muitos dos diagnósticos feitos apenas pelo EC. Por exemplo, no diagnóstico de uma regurgitação mitral crónica a auscultação pode ser descrita como “...um sopro holosistólico de grau 3 na área mitral, com irradiação axilar e incorporando o $1^{\circ}$ tom cardíaco, combinado com um rodado mesodiastólico antecedido por um $3^{\circ}$ tom cardíaco". Mas um simples exame ecocardiográfico dar-nos-á - entre outras medições - a superfície valvular, as dimensões das cavidades cardíacas, assim como a fracção de ejecção

*Centro de Estudos de Medicina Baseada na Evidência Cochrane Portugal Faculdade de Medicina da Universidade de Lisboa do ventrículo esquerdo e respectivo volume de refluxo sistólico. Dificilmente se poderá ter melhor!

Esta nova realidade fez com que houvesse a ideia de que o EC clássico teria perdido muita da sua validade, podendo ser substituído pela tecnologia, num processo combinando alguma informação do EC com dados provenientes de meios auxiliares de diagnóstico (raio $\mathrm{X}$ na pneumonia, TC no AVC, etc.).

Ora esta abordagem é errada, já que o EC se revela de grande importância como base inicial do processo diagnóstico. ${ }^{2}$ Na maior parte dos casos, um diagnóstico de qualidade assenta na informação obtida através do EC, permitindo colocar o doente em determinado grupo de probabilidade para uma doença específica: se um sintoma ou sinal típico dessa patologia está presente (achado positivo), o diagnóstico é mais provável. Se, por outro lado, os sintomas e sinais típicos estão ausentes - achados negativos - então a probabilidade de doença diminui. Como se verá mais adiante, este passo é absolutamente crucial.

\section{ABORDAGEM PROBABILÍSTICA DE DOENÇA UTILIZANDO SINTOMAS E SINAIS DO EXAME CLÍNICO}

O impacto dos achados positivos (e negativos) no diagnóstico de doença varia dependendo da capacidade de cada um deles alterar a chamada probabilidade pré-teste, que se define como a probabilidade de doença antes da efectivação do EC. Por outras palavras, é a prevalência. $^{3}$

A importância da prevalência no diagnóstico é exemplificada nos seguintes casos clínicos:

1. Uma jovem de 22 anos, perfeitamente saudável, vem ao serviço de urgência com um quadro de dor retrosternal (DRE) típica de isquémia aguda do miocárdio. 
2. Homem de 65 anos, obeso, fumador, hipertenso e diabético, vem ao serviço de urgência com um quadro de DRE típica de isquémia aguda do miocárdio.

Estudos determinaram que as características da DRE típica aumentam 35\% as hipóteses diagnósticas de isquémia aguda do miocárdio. Aplicando esta informação aos dois casos, os resultados são radicalmente diferentes: no primeiro caso, como a prevalência/probabilidade pré-teste é de apenas $1 \%$, este sintoma sobe a probabilidade de doença para $36 \%(35+1)$, insuficiente para um diagnóstico provisório. No segundo caso (homem de 65 anos), a probabilidade pré-teste é de $60 \%$, pelo que a presença da DRE aumenta a probabilidade de EAM para $95 \%$ (60+35), um resultado praticamente diagnóstico.

Como se pode ver, os sintomas e sinais comportam-se-em termos das suas capacidades discriminatórias - exactamente como os resultados de testes auxiliares de diagnóstico e devem ser interpretados analogamente. O cálculo das características diagnósticas de um achado no EC está representado no Quadro I, utilizando o exemplo da regurgitação mitral.

Os valores mais importantes a levar em linha de conta nos dados do EC são a sensibilidade (a proporção de doentes que apresentam o sintoma ou sinal específicos - taxa de verdadeiros positivos), a especificidade (a proporção de saudáveis que estão assintomáticos ou que não apresentam o sinal específico - taxa de verdadeiros negativos), o valor preditivo positivo (se o sintoma e/ou sinal está presente, qual é a probabilidade de o paciente ter a doença), o valor preditivo negativo (se o sintoma e/ou sinal está ausente, qual é a probabilidade de o paciente não ter a doença), o likelihood ratio positivo (que é a proporção de doentes apresentando o sintoma ou sinal, dividido pela proporção de saudáveis que também apresentam o mesmo sintoma ou sinal) e o likelihood ratio negativo (que é a proporção de doentes que não apresentam o sintoma ou sinal, dividido pela proporção de saudáveis que também não apresentam o mesmo sintoma ou sinal).

No processo diagnóstico, o mais importante são os likelihood ratios (LRs), que permitem calcular a hipótese pós-teste segundo a fórmula "Probabilidade pós-teste = probabilidade pré-teste x LR” (ver os dois exemplos apresentados).

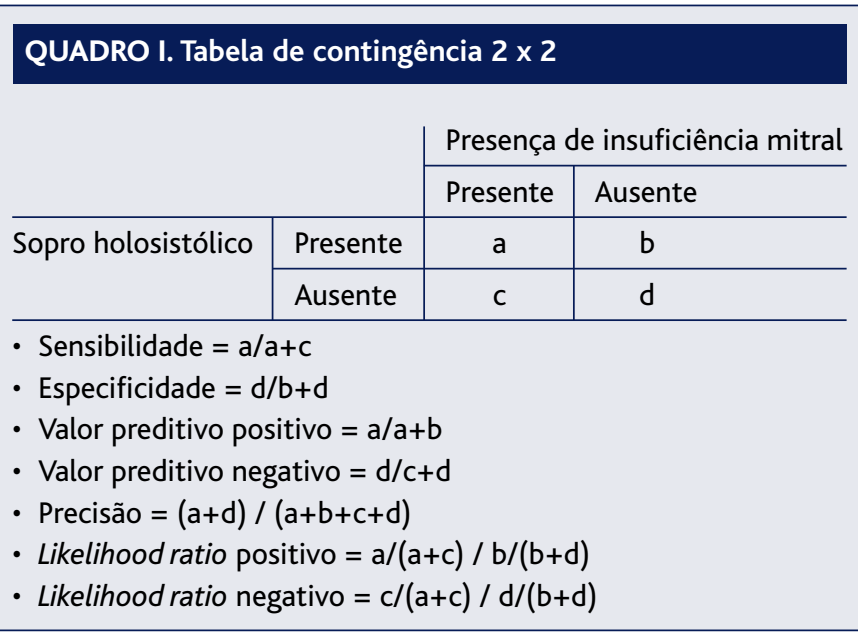

\begin{tabular}{|l|c|}
\hline \multicolumn{2}{|c|}{ QUADRO II. Likelihood ratios e estimativas de doença } \\
\hline Likelihood ratio & Alterações na probabilidade de doença \\
\hline 0,1 & $-45 \%$ \\
0,2 & $-30 \%$ \\
0,3 & $-25 \%$ \\
0,5 & $-15 \%$ \\
1 & $0-$ sem alteração \\
2 & $+15 \%$ \\
3 & $+20 \%$ \\
4 & $+25 \%$ \\
5 & $+30 \%$ \\
8 & $+40 \%$ \\
10 & $+45 \%$ \\
\hline
\end{tabular}

Porque os médicos necessitam de uma medida de precisão diagnóstica de cada achado do EC, os LRs de cada sintoma ou sinal (isolado ou em combinação sindromática) fornecem exactamente essa medida. Cada um está associado a um LR (positivo ou negativo) que varia entre 0 e infinito: os superiores a 1 aumentam a probabilidade de doença, os inferiores a 1 diminuem essa probabilidade e os próximos da unidade não a alteram significativamente (isto é, são inúteis para o diagnóstico).

Uma das maneiras práticas de utilizar os LRs é a de calcular as mudanças nas probabilidades de doença baseadas nos seus valores (Quadro 2). 


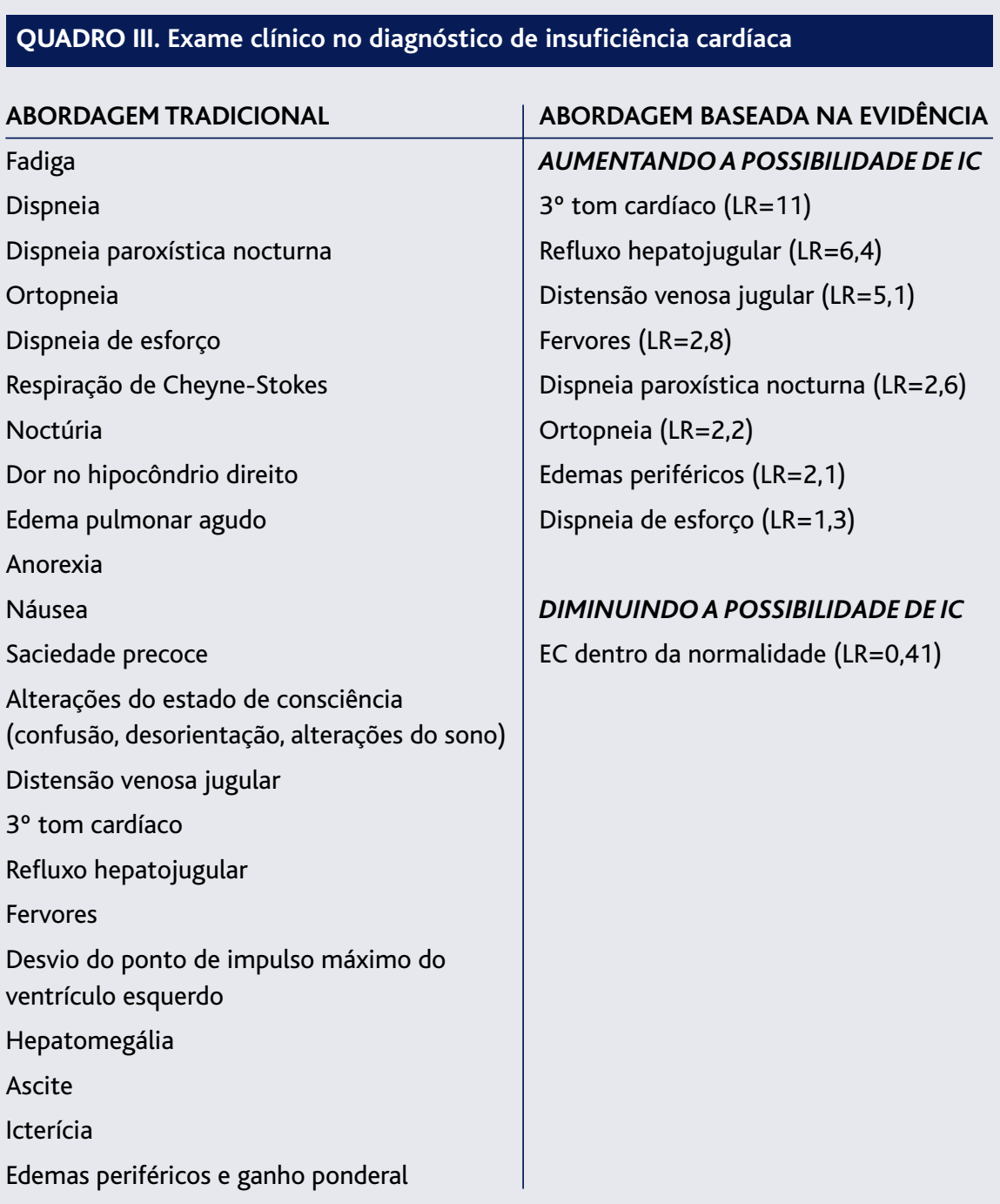

guntar e como integrar a informação obtida na obtenção de um diagnóstico final coerente.

Por outro lado, se consultarmos dois estudos definindo o diagnóstico baseado na evidência dos achados do EC, ${ }^{4-5}$ podemos identificar uma lista mais curta e selectiva de sintomas e sinais preditivos da IC, que estão na coluna da direita do Quadro 3. Utilizando, nomeadamente no contexto do serviço de urgência, os sintomas e sinais referidos, poderemos ter uma outra confiança no diagnóstico de IC num doente que se apresenta com dispneia (por exemplo) e que não sabemos se tem por hipótese DPOC. Esta abordagem permite diminuir a incerteza dos achados do EC e chegar mais consistentemente a uma hipótese pré-teste de doença.

Existe hoje publicada informação sobre os LRs dos achados do EC numa quantidade muito vasta de doenças, sendo a série da Rational Clinical Examination, do JAMA, a mais conhecida ${ }^{6}$

\section{EXEMPLO DE UMA ABORDAGEM DIAGNÓSTICA NA INSUFICIÊNCIA CARDÍACA}

Os doentes com insuficiência cardíaca (IC) apresentam uma semiologia muito rica, reflectindo a complexidade fisiopatológica desta patologia.

Se consultarmos o capítulo sobre Heart failure, do Harrison's (19 ${ }^{\text {th }}$ edition, 2015), vemos que se encontram descritos um conjunto vasto de sintomas e sinais, mas sem qualquer hierarquização em termos de capacidade discriminatória para o diagnóstico final (coluna da esquerda do Quadro 3). Ou seja, a listagem de achados do EC não está ordenada em termos de poder suportar (ou eliminar) a possibilidade de estarmos perante um doente com IC, pelo que ficamos na dúvida sobre que per-

\section{CONCLUSÕES}

O EC está a ser sujeito aos mesmos critérios exigentes que qualquer outra tecnologia laboratorial ou de imagem, nomeadamente através de estudos que comparam os achados da história e exame objectivo com um gold standard considerado diagnóstico de uma determinada doença.

Ao compararmos a presença de sintomas e sinais em doentes com os não doentes obtemos uma mediada de associação - o likelihood ratio - que nos permite seleccionar os que mais utilidade têm para o diagnóstico provisório.

Estes estudos obedecem aos critérios STARD, ${ }^{7-8}$ definindo rigorosamente o sintoma/sinal e os critérios 
diagnósticos normalizados em doentes com sintomas sugestivos da doença em estudo, com ocultação contra o teste de comparação e com dados em quantidade suficiente para permitir o cálculo das medidas de associação relevantes para a sua operacionalização: sensibilidade, especificidade, valores preditivos e, acima de tudo, os likelihood ratios.

A nova era do exame clínico irá basear-se em dados de prevalência de doença facilmente adquiríveis, incorporará dados discriminatórios científicos dos achados da colheita da história e do exame físico e combiná-los-á para obtenção de uma probabilidade de doença e subsequente selecção dos meios auxiliares de diagnósticos (sujeitos ao mesmo rigoroso escrutínio científico).

\section{REFERÊNCIAS BIBLIOGRÁFICAS}

1. McGee S. Evidence-based physical diagnosis. 3rd ed. Philadelphia:W.B. Saunders; 2012. ISBN 9781437722079

2. Verghese A, Horwitz RI. In praise of the physical examination. BMJ. 2009;339:b5448.

3. Guyatt G, Rennie D, Meade MO, Cook DJ. Users' guides to the medical literature: a manual for evidence-based clinical practice. 3rd ed. New York: McGraw-Hill; 2015. ISBN 9780071790710

4. Badgett RG, Lucey CR, Mulrow CD. Can the clinical examination diagnose left-sided heart failure in adults? JAMA. 1997;277(21):1712-9.

5. Wang CS, FitzGerald JM, Schulzer M, Mak E, Ayas NT. Does this dyspneic patient in the emergency department have congestive heart failure? JAMA. 2005;294(15):1944-56.

6. Ebell MH, Call M, Shinholser J, Gardner J. Does this patient have infectious mononucleosis? The rational clinical examination systematic review. JAMA. 2016;315(14):1502-9.

7. Bossuyt PM, Reitsma JB, Bruns DE, Gatsonis CA, Glasziou PP, Irwig LM, et al. Towards complete and accurate reporting of studies of diagnostic accuracy: the STARD initiative. Ann Intern Med. 2003;138(1):40-4.

8. Bossuyt PM, Reitsma JB, Bruns DE, Gatsonis CA, Glasziou PP, Irwig L, et al. STARD 2015: an updated list of essential items for reporting diagnostic accuracy studies. BMJ. 2015;351:h5527.

\section{CONFLITO DE INTERESSES}

O autor declara não ter conflitos de interesses.

\section{ENDEREÇO PARA CORRESPONDÊNCIA}

E-mail: avc@medicina.ulisboa.pt

Artigo redigido ao abrigo do acordo ortográfico anterior a 1990. 